W stużbie tradycji i odnowy liturgicznej. 50 lat Instytutu Liturgicznego w Krakowie (1968-2018), red. P. Nowakowski, J. Mieczkowski, Kraków 2019, s. 263-271.

ISBN 978-83-7438-849-8 (wersja drukowana), ISBN 978-83-7438-850-4 (wersja online) DOI:http://dx.doi.org/10.15633/9788374388504.20

\title{
Das Deutsche Liturgische Institut in Trier Seine Bedeutung für die liturgische Erneuerung und seine heutigen Aufgaben
}

Es war im Jahr 1917, als sich ein junger Priester der Diözese Mainz nach einem Besuch der Benediktinerinnenabtei St. Hildegard in Eibingen und dem Miterleben der Liturgie einer Jungfrauenweihe Gedanken machte, was denn Liturgie eigentlich ist. Aus diesen Aufsätzen mit Überschriften wie „Liturgische Gemeinschaft" oder „Liturgie als Spiel“ wurde ein berühmtes und folgenschweres Büchlein, das im folgenden Jahr 1918 - also vor 100 Jahren - unter dem Titel Vom Geist der Liturgie erstmals erschien und seitdem unzählige Auflagen in verschiedenen Sprachen erlebt hat. Im Laufe dieses Jahres 2018 wurde in Deutschland an zahlreichen Orten mit Tagungen und Vorträgen an diese Jahrhundertschrift und ihren Autor Romano Guardini erinnert, vor allem an dessen Wirkungsstätten. Eine Wanderausstellung, die vom Abt-Herwegen-Institut der Abtei Maria Laach konzipiert wurde, zeichnete die Entstehung dieses Werkes nach; sie wurde $u$. a. in Maria Laach, Trier, Burg Rothenfels, Köln und München präsentiert. ${ }^{1}$

1 Cf. S. K. Langenbahn, Vom Geist der Liturgie. 100 Jahre Romano Guardinis "Kultbuch" der Liturgischen Bewegung, Köln 2017; M. Linnenborn, 100 Jahre „Vom Geist der Litur- 
Vom Geist der Liturgie wirkte wie eine Initialzündung für die Liturgische Bewegung in Deutschland. Durch die Jugendbewegung und junge Priester, die an der Erneuerung der Liturgie besonders interessiert waren, verbreiteten sich die Gedanken der Liturgischen Bewegung. Die liturgischen Erneuerungsbestrebungen fanden aber nicht nur Zustimmung, sondern auch harsche Kritik und Ablehnung. Um diese Auseinandersetzungen zu kanalisieren, berief die Deutsche Bischofskonferenz im Jahr 1940 zwei Bischöfe $^{2} \mathrm{zu}$ „Referenten für liturgische Angelegenheiten“ und eine „Liturgische Kommission“ als deren Beratergruppe. Zu diesen Beratern gehörte auch Romano Guardini. Die Liturgische Kommission erhielt ein Sekretariat in Trier, da der damalige Trierer Generalvikar Heinrich von Meurers ${ }^{3}$ die finanziellen Mittel dafür zur Verfügung stellte. Sekretär wurde der junge Trierer Diözesanpriester Dr. Johannes Wagner (1908-1999).

Nach dem Ende des Weltkriegs wuchs der Wunsch nach einer Arbeitsstelle, die sich auch unterhalb der offiziellen Ebene der „Liturgischen Kommission" der Bischofskonferenz für die Erneuerung der Liturgie einsetzen konnte. Es ging schließlich nicht nur um kirchenamtliche Fragen, sondern auch um Publikationen für die liturgische Praxis, die Veranstaltung von Tagungen und Kursen sowie natürlich um eine breite Liturgische Bildung. So kam es 30 Jahre, nachdem sich Guardini seine Gedanken über den Geist der Liturgie gemacht hatte, am Ende des Jahres 1947 zur Gründung einer solchen Arbeitsstelle "zur Förderung des Liturgischen Apostolats“, des heutigen Deutschen Liturgischen Instituts, das wiederum in Trier seinen Sitz hat, da sich das Bistum Trier weiterhin dem Anliegen der liturgischen Erneuerung verpflichtet wusste. Zur Diözese Trier gehört die Abtei Maria Laach, einer der Ursprungsorte der Liturgischen Bewegung.

So war es sicher kein Zufall, dass in derselben Sitzung der Liturgischen Kommission am 17. Dezember 1947 in Augsburg, in der man über die kurz zuvor veröffentlichte Enzyklika Papst Pius' XII. Mediator Dei beriet, auch die Gründung des Liturgischen Instituts beschlossen wurde. Zu den

gie“. Eine Wanderausstellung zum Kultbuch der Liturgischen Bewegung, "Gottesdienst" 52 (2018), S. 96.

2 Bischof Albert Stohr von Mainz und Bischof Simon Landersdorfer von Passau.

3 Cf. G. Pasenow, Heinrich von Meurers (1888-1953). Ein Förderer und Wegbereiter der liturgischen Erneuerung in Deutschland (TThSt 80), Trier 2016, besonders 486-492. 
Unterzeichnern des Gründungsprotokolls unseres Instituts gehört wiederum auch Romano Guardini, der in demselben Jahr 1947 der gefeierte Redner beim Zweiten Liturgischen Kongress Frankreichs in Lyon war. ${ }^{4}$ Die Enzyklika Mediator Dei gab womöglich den letzten Ausschlag, den schon seit längerer Zeit gehegten Wunsch nach einer eigenständigen Arbeitsstelle zur Förderung und Koordinierung des liturgischen Apostolats in Deutschland nun in die Praxis umzusetzen. ${ }^{5}$ Johannes Wagner, Sekretär der Liturgischen Kommission, wurde erster Leiter des Instituts (im Amt bis 1975). Ihm gelang es,

aus bescheidensten Anfängen das Liturgische Institut kontinuierlich zu einem weit über die deutschen Grenzen hinaus bekannten Zentrum pastoralliturgischer Initiativen und liturgiewissenschaftlicher Forschung auszubauen. ${ }^{6}$

Eine weitere bedeutende Persönlichkeit des Liturgischen Instituts trat in demselben Jahr in die Öffentlichkeit: Balthasar Fischer (1912-2001) war im Mai 1947 zum Professor für Liturgiewissenschaft am Priesterseminar in Trier ernannt worden. Als im Jahr 1950 die Theologische Fakultät in Trier wiederbegründet wurde, wurde mit dem Lehrstuhl für Liturgiewissenschaft der erste dieses Faches in Deutschland errichtet und Balthasar

4 Die weiteren Unterzeichner sind: Heinrich von Meurers, Theodor Klauser, Theodor Schnitzler, Theodor Bogler OSB, Johannes Wagner, Klemens Tillmann, Heinrich Kahlefeld, Ludwig Wolker.

5 Im Gründungsprotokoll heißt es: „[...] bewegt von dem Wunsche, das liturgische Apostolat im Sinne der eben zur Kenntnis gelangten Enzyklika Pius' XII. ,Mediator Dei' vom 20.11.47 in den deutschen Diözesen zu fördern, schließen sich die Unterzeichneten zusammen zur Gründung eines Liturgischen Institutes [...]. Zweck des Institutes ist: Förderung des Liturgischen Apostolates in seinem ganzen Umfang, insbesondere der praktischen gottesdienstlichen Arbeit. Mittel zur Erreichung des Zieles sind: Wissenschaftliche Klärung liturgischer Fragen, Beratung der Seelsorger, Bereitstellung zuverlässigen Arbeitsmaterials, besonders geeigneten Schrifttums und eines Organs, Abhaltung von Kursen, Tagungen, Konferenzen, Kongressen.“

6 A. Heinz, Das Bistum Trier und das Zweite Vatikanische Konzil, in: A. Heinz, Liturgie und Frömmigkeit. Beiträge zur Gottesdienst- und Frömmigkeitsgeschichte des (Erz-)Bistums Trier und Luxemburgs zwischen Tridentinum und Vatikanum II, Trier 2008, S. 463. 
Fischer zum Professor ernannt. Als Leiter der Wissenschaftlichen Abteilung des Liturgischen Instituts war er in dessen Arbeit voll integriert. Damit sind die beiden wichtigsten Namen genannt, die nicht nur in den folgenden Jahrzehnten die Arbeit in Trier prägten, sondern auch eine wichtige Rolle beim Konzil und der folgenden Erneuerung der Liturgie spielten.

Einer breiteren kirchlichen Öffentlichkeit wurde das Liturgische Institut im Jahr 1950 durch den Ersten Deutschen Liturgischen Kongress in Frankfurt bekannt, der von Trier aus organisiert wurde zum Thema Die Eucharistiefeier am Sonntag - eine Thematik, die auch im Mittelpunkt der zwei schon einige Jahre zuvor stattgefundenen Nationalen Liturgischen Kongresse in Frankreich stand. ${ }^{7}$

In den Jahren vor dem Konzil spielten die internationalen Kontakte, die vom Liturgischen Institut organisiert wurden, eine wichtige Rolle bei der Vorbereitung der späteren Liturgiereform. In Kooperation mit dem Centre de pastorale liturgique in Paris organisierte das Trierer Institut in den Jahren zwischen 1951 und 1960 an verschiedenen Orten Europas sieben Internationale Liturgische Studientreffen. Das erste Treffen dieser Reihe fand im Juli 1951 in Maria Laach statt.

Die bei dem Laacher Treffen formulierten Reformwünsche hinsichtlich der Messfeier enthielten schon fast alles, was diesbezüglich später in die Liturgiekonstitution hineingeschrieben wurde. ${ }^{8}$

Mit diesen Studientreffen, deren Themen bereits die Fragen einer möglichen zukünftigen Liturgiereform bildeten - Reform der Messe und der Perikopenordnung, Wiederherstellung der Konzelebration, Reform des Breviers, Initiation von Erwachsenen und Kindern - wurden in enger

7 Von den vier bei dem Kongress verabschiedeten Resolutionen wurden drei schon bald von Rom gestattet: Wiederherstellung der Osternacht, Abendmessen, Milderung des Nüchternheitsgebotes. Die vierte Resolution, die Verkündigung von Lesung und Evangelium in der Volkssprache, wurde durch das Konzil verwirklicht. Cf. A. Heinz, Das Bistum Trier und das Zweite Vatikanische Konzil, S. 463.

8 A. Heinz, Die Bedeutung des Deutschen Liturgischen Instituts in Trier im Kontext der Liturgiereform des Zweiten Vatikanischen Konzils, "Quellen und Abhandlungen zur Mittelrheinischen Kirchengeschichte“ 137 (2015), S. 143. 
Zusammenarbeit zwischen deutsch- und französischsprachigen Liturgiewissenschaftlern wichtige Weichen für die Erneuerungsarbeit des Konzils gelegt, von dem zu diesem Zeitpunkt noch niemand wusste, dass es in wenigen Jahren bevorsteht.

Bereits in der Zeit der unmittelbaren Vorbereitung des Zweiten Vatikanischen Konzils gehörten Johannes Wagner und Balthasar Fischer zu den Konsultoren der Liturgischen Vorbereitungskommission. Hier konnten sie eng zusammenarbeiten mit dem Innsbrucker Liturgiewissenschaftler Josef Andreas Jungmann SJ, der selbst Mitglied der Kommission war. Wagner wurde dann auch zum Peritus der Konzilskommission für die Liturgie berufen. Während des Konzils wurde Balthasar Fischer als Peritus seines Trierer Bischofs Matthias Wehr hinzugezogen. Das Votum der Trierer Fakultät bildete das umfangreichste und inhaltlich gewichtigste unter den von den katholischen Hochschulen im Vorfeld des Konzils eingereichten Reformvorschlägen zur Liturgie.

Wagner und Fischer wurden schließlich zu Konsultoren des Consilium ad exsequendam Constitutionem de sacra Liturgia, das mit der Durchführung der Konzilsbeschlüsse beauftragt wurde, und später auch zu Konsultoren der Gottesdienstkongregation ernannt. Wagner moderierte als Relator den wichtigen Coetus X Ordo Missae, Fischer die Arbeitsgruppe zur Reform der Taufliturgie. ${ }^{9}$

Die Messreform sollte für den Leiter des deutschen Liturgischen Instituts der Auftrag seines Lebens werden. Für immer wird Wagners Name mit dem 1970 eingeführten Missale Romanum Pauls VI. verknüpft sein. ${ }^{10}$

In der Zeit nach dem Konzil kamen dem Liturgischen Institut in Trier für die Rezeption der Liturgiekonstitution Sacrosanctum Concilium drei wichtige Aufgabenbereiche zu: die Vermittlung der zentralen Anliegen der Liturgiekonstitution, die Bereitstellung der volkssprachlichen Liturgiebücher und die Förderung der liturgischen Bildung im Geist der erneu-

9 Balthasar Fischers Expertise war außerdem besonders gefragt zu den Themen Konzelebration, Psalmenverteilung im Stundenbuch, Erneuerung der Initiationsliturgie, später auch zum Direktorium für Messfeiern mit Kindern und zu den Kinder-Hochgebeten.

1o A. Heinz, Das Bistum Trier und das Zweite Vatikanische Konzil, S. 473. 
erten Liturgie. Diesen Anliegen dienen zwei regelmäßige Publikationen, die vom Institut herausgegeben werden: das liturgiewissenschaftliche Periodikum „Liturgisches Jahrbuch“ (seit 1951) und die alle zwei Wochen erscheinende Zeitschrift „,Gottesdienst“ (seit 1967), die gemeinsam mit den Liturgischen Instituten Österreichs und der deutschsprachigen Schweiz verantwortet wird. Der tieferen Durchdringung der Liturgiekonstitution dienten auch große Tagungen: die Liturgischen Kongresse 1964 in Mainz und 1965 in Berlin (Ost). Von 1965 bis 1975 veranstaltete das Institut die "Trierer Liturgiewissenschaftlichen Studienkurse“, eine jeweils einjährige liturgiewissenschaftliche Spezialausbildung, die Priester und Laien als Multiplikatoren für die praktische liturgische Arbeit in Diözesen und Ordensgemeinschaften schulen wollte. Der weitaus größere Teil der Teilnehmer an den Studienkursen kam von außerhalb des deutschen Sprachraumes, sodass durch diese Internationalität von Trier aus wichtige Impulse auch in andere Länder und Sprachräume ausgingen. ${ }^{11}$ Die Aufgabe der Fortbildung übernimmt seit 1985 das Angebot „Liturgie im Fernkurs“, an dem derzeit rund 120 haupt- und ehrenamtlich in Pfarreien tätige Frauen und Männer über mehrere Jahre mit Lehrbriefen und Studienwochenenden teilnehmen; eine Prüfung kann als Voraussetzung für die Übernahme bestimmter liturgischer Dienste dienen, z. B. die Leitung von Wort-Gottes-Feiern oder den Begräbnisdienst. Seit 2002 veranstaltet das Institut jährlich die „Trierer Sommerakademie Liturgie“", an der regelmäßig rund 50 haupt- und ehrenamtlich an liturgischen Fragen Interessierte teilnehmen. 2018 war das Thema Liturgie und Bibel, 2019 wird sich die Tagung mit Liturgie und Lebensraum beschäftigen; die Vorträge der Sommerakademie werden jeweils in Dokumentationsbänden publiziert. Das Institut gibt eine Vielzahl von pastoralliturgischen Hilfen und Materialien heraus, die gottesdienstliche Praxis in den Pfarreien unterstützen.

Als größte Einrichtung im deutschen Sprachgebiet übernimmt das Deutsche Liturgische Institut seit Beginn der Umsetzung der Liturgiereform die Federführung bei der Erarbeitung und schließlich auch die Redaktion der offiziellen deutschsprachigen liturgischen Bücher. Die Übersetzungsarbeit

11 Von den insgesamt 143 Teilnehmern kamen nur 40 aus den deutschsprachigen Ländern; vgl. A. Waibel, Die Studienkurse des Deutschen Liturgischen Instituts in Trier, "Liturgisches Jahrbuch“ 40 (1990), S. 211-227. 
selbst übernehmen jeweils Kommissionen von Professoren und weiteren Fachleuten aus den deutschsprachigen Ländern. So konnte in den Jahren von 1970 bis 1976 die erste Generation der deutschsprachigen Liturgiebücher erscheinen. Bis heute bildet diese Aufgabe einen der Schwerpunkte in der Arbeit des Instituts. Die Übernahme der 2016 erschienenen revidierten Einheitsübersetzung der Heiligen Schrift in die verschiedenen liturgischen Bücher wird mehrere Jahre in Anspruch nehmen; zum Beginn des neuen Kirchenjahrs ist kürzlich der erste Band des Lektionars für die Sonntage und Festtage im Lesejahr C erschienen. Zu Recht wurde unser Institut einmal als die „wichtigste Werkstatt für die Umsetzung der Liturgiereform im deutschen Sprachgebiet ${ }^{\prime \prime 12}$ bezeichnet.

Die Funktion unseres Instituts lässt sich heute wohl am treffendsten beschreiben als eine Mischung von wissenschaftlichem und pastoralliturgischem Zentrum und einem liturgischen Büro für Deutschland und die übrigen deutschsprachigen Länder. In unserem Haus gibt es eine Bibliothek mit mehr als 80.0oo Bänden aus den verschiedenen Sprachräumen, die von den Experten, die sie regelmäßig nutzen, als die größte Bibliothek für Liturgiewissenschaft überhaupt bezeichnet wird. Unser Archiv bewahrt u. a. die Nachlässe bedeutender Liturgiewissenschaftler ${ }^{13}$ sowie wertvolle Dokumente des Zweiten Vatikanischen Konzils und der Umsetzung der Liturgiereform auf - zum Beispiel eine große Zahl der Schemata der Liturgiekonstitution, die in Rom aufgrund der Sperrfrist noch nicht einsehbar sind - und bietet damit eine einmalige zeitgeschichtliche Dokumentation über das Konzil und seine Wirkungsgeschichte.

Das Deutsche Liturgische Institut arbeitet in enger Vernetzung mit den Liturgischen Instituten Österreichs und der deutschsprachigen Schweiz und pflegt gute Kontakte zu Einrichtungen und Gremien im Bereich der Ökumene. Mitarbeiter des Instituts halten als Mitglieder oder ständige Gäste in verschiedenen Konferenzen den Kontakt zu den Bereichen Kirchenmusik, Kunst und Kirchenbau, Glockenwesen. Das Institut selbst lädt die Liturgiereferate der deutschen Diözesen, die Liturgikdozenten in der

\footnotetext{
12 A. Heinz, Das Bistum Trier und das Zweite Vatikanische Konzil, S. 475.

13 Balthasar Fischer, Emil Joseph Lengeling, Heinrich Rennings (Leiter des DLI von 1984 bis 1995), Johannes Wagner.
} 
Priesterausbildung und die Diözesanverantwortlichen für Kinder- und Familiengottesdienste zu jährlichen Vernetzungstreffen ein.

Das Deutsche Liturgische Institut versteht sich in der Funktion eines Kundschafters, der beobachtet, welche Themen und Fragen in der Luft liegen, sowie als Dienstleister und Impulsgeber für das gottesdienstliche Leben vor Ort. Diese Aufgaben können nur in enger Kooperation und Vernetzung mit allen, die im liturgischen Bereich wissenschaftlich und praktisch tätig sind, erfüllt werden. So steht unser Institut an verschiedenen Schnittstellen: von Wissenschaft und Pastoral, von Ortskirche und Weltkirche, von kirchlichen Institutionen und Pfarrgemeinden. Letztlich dient alles dem Ziel, das Verständnis der Liturgie zu vertiefen und das gottesdienstliche Leben vor Ort zu stärken und zu verlebendigen, damit die Kirche bei allen Veränderungen und Herausforderungen auch in Zukunft ecclesia orans bleibt, in der die Menschen die Gegenwart Gottes erfahren und ihn mit ihrem ganzen Leben loben können, gemeinsam mit den Schwestern und Brüdern in der Gemeinschaft der Kirche. Denn, so formulierte es Guardini in Vom Geist der Liturgie, die „Liturgie sagt nicht 'Ich', sondern, ‘Wir'“'14 - was könnte aktueller sein für unsere Zeit?

\section{Streszczenie}

Historia i znaczenie Instytutu Liturgicznego w Trewirze podczas odnowy liturgicznej oraz jego dzisiejsza rola w krajach niemieckojezycznych

Niemiecki Instytut Liturgiczny (DLI) w Trewirze został założony w 1947 roku przez kilku księży, aby promować apostolstwo liturgiczne oraz pracować na rzecz rozwoju i propagowania idei ruchu liturgicznego. Od dnia założenia jego siedziba znajduje się w Trewirze. Wsród założycieli był Romano Guardini, jeden z najważniejszych krzewicieli odnowy liturgicznej. Od roku 1947 w Trewirze znajduje się pierwsza katedra nauk liturgicznych w zakresie języka niemieckiego; pierwszym profesorem był Balthasar Fischer (1912-2001). W latach przed soborem watykańskim II, podczas niego i po jego zakończeniu Niemiecki Instytut Liturgiczny osiągnął

14 R. Guardini, Vom Geist der Liturgie, Freiburg im Breisgau 1983, S. 45. 
swoje największe znaczenie. Dyrektor Instytutu Johannes Wagner (1908-1999) był relatorem zespołu Coetus X ds. reformy Ordo Missae. Ścisły związek między instytutami w Trewirze i w Paryżu wniósł wielki wkład w odnowę liturgiczną oraz $\mathrm{w}$ realizację reformy liturgicznej.

Dziś DLI składa się z trzech wielkich części: z urzędu celebracji liturgicznych Konferencji Episkopatu Niemiec, który wykonuje również zadania dla wszystkich krajów niemieckojęzycznych, zwłaszcza redaguje księgi liturgiczne; z części liturgiczno-duszpasterskiej zajmującej się publikacją czasopisma "Gottesdienst" oraz różnych materiałów pomocniczych do celebracji i formacji liturgicznej; z części naukowo-badawczej z ogromną biblioteką (około 80 tys. tomów) i archiwum, w którym przechowuje się dorobek naukowy kilku ważnych liturgistów oraz publikuje czasopismo „Liturgisches Jahrbuch”. 
